\title{
Refractive index sensitivity of Au nanostructures in solution and on the substrate
}

\author{
Hemant Ramakant Hegde ${ }^{1}$ (D), Santhosh Chidangil ${ }^{1}$, and Rajeev K. Sinha ${ }^{1, *}$ \\ ${ }^{1}$ Department of Atomic and Molecular Physics, Manipal Academy of Higher Education, Manipal, Karnataka 476104, India
}

Received: 29 October 2021

Accepted: 12 December 2021

Published online:

25 December 2021

(C) The Author(s) 2021

\begin{abstract}
In this work, we present the synthesis and surface immobilization of $\mathrm{Au}$ nanostars, $\mathrm{Au}$ nanocubes and $\mathrm{Au}$ nanorods for localized surface plasmon resonance (LSPR)-based refractometric sensing applications. Au nanostructures exhibiting LSPR peak positions in 500-900 $\mathrm{nm}$ spectral range were prepared by seed-mediated synthesis method. The refractive index (RI) sensitivity of all these nanostructures in the colloidal solution were measured and the sample exhibiting highest sensitivity in each category were immobilized on the glass substrate. The surface immobilized nanostructures were investigated for RI sensing. Au nanostars having LSPR peak position at $767 \mathrm{~nm}$ exhibited highest RI sensitivity of $484 \mathrm{~nm} /$ RIU in solution and $318 \mathrm{~nm} /$ RIU on the substrate. This study gives an outline for selecting the $\mathrm{Au}$ nanostructures for developing plasmonic sensing platforms.
\end{abstract}

\section{Introduction}

Noble metal nanostructures support localized surface plasmon resonance (LSPR), a phenomenon in which the conduction electrons present on the surface of the nanostructures exhibit coherent oscillations when electromagnetic radiation of suitable frequency interacts with them [1]. One or more extinction bands are observed in near-UV-Vis-NIR region of the electromagnetic spectrum due to plasmonic resonance [2]. The plasmonic resonant peaks are sensitive to the surrounding dielectric environment and show redshift when the refractive index (RI) of the surrounding medium is increased. This RI sensing

Address correspondence to E-mail: rajeev.sinha@manipal.edu property of plasmonic nanostructures is utilized in the construction of LSPR-based refractometric sensors [3]. The RI sensitivity is expressed as the shift in LSPR peak position per unit change in RI of the surrounding medium. The RI sensitivity of the plasmonic nanostructures is determined by the material composition, shape and size of the nanostructures [4]. Silver and gold are the most popular plasmonic materials and gold being relatively inert and stable, gained popularity in various sensing applications [5]. Highly symmetric spherical nanoparticles exhibit single peak in extinction spectrum whereas anisotropic nanostructures like nanorods show multiple peaks due to polarization of free electrons at the 
corners and edges. Thus the optical properties of plasmonic metal nanostructures can be tuned by controlling the shape [6]. Shape of the nanocrystals also play most important role in determining the RI sensitivity and anisotropic nanostructures exhibit higher sensitivity compared to spherical nanoparticles [7]. Plasmonic nanostructures with sharp tips exhibit higher sensitivity compared to nanostructures with similar aspect ratios [7]. For a metal nanostructure of given material composition and shape, size is the most important parameter which determines the sensitivity. So evaluating the sensitivity of nanoparticles of different sizes for a given shape gives the guidelines for selecting a nanostructure for specific applications.

Increased need for label free sensing techniques, multiplexing and miniaturization are offering great prospect for substrate-based plasmonic sensing. Solution phase chemical synthesis gives relatively low cost convenient method for fabrication of nanostructures and the chemically synthesized nanostructures can be effectively surface immobilized. Tremendous developments in the surface modification techniques of plasmonic nanostructures are boosting the possibility of development of state of the art sensing platforms [8, 9]. The response of plasmonic nanostructures to the small changes in the RI of the surrounding medium has made them very attractive for signal transducers in refractometric detection. Au nanostars, nanocubes and nanorods having sharp edges exhibit intense electromagnetic field enhancement which make them very attractive for sensing applications. Chen et al. investigated the RI sensitivity of gold nanospheres, nanocubes, nanobranches, nanorods, and nanobipyramids and reported that nanobranches exhibited highest sensitivity of $703 \mathrm{~nm} /$ RIU [10]. Gold nanostars and nanocubes exhibited RI sensitivity of 326 and $83 \mathrm{~nm} /$ RIU, respectively [10, 11]. Gold nanorods exhibited sensitivity of $252 \mathrm{~nm} / \mathrm{RIU}$ after immobilization on glass substrate [12]. Several computational studies have been done on the RI sensitivity of different anisotropic $\mathrm{Au}$ nanostructures [13-15]. Since the theoretical study gives an upper bound to the sensitivity value, it is desirable to study the RI sensitivity of $\mathrm{Au}$ nanostructures experimentally, while selecting them for sensor development. The colloidal suspension of nanoparticles are not thermodynamically stable, change in $\mathrm{pH}$, ionic strength and temperature affect the nanoparticles in the solution. The nanoparticles can be easily aggregated in solution phase. Immobilization of nanostructures on the substrate effectively prevents the aggregation and facilitates the multiple usage of substrates [16]. The substrate immobilized nanostructures can be easily functionalized and can be integrated with microfluidic systems. Electromagnetic field around the plasmonic nanostructures changes considerably after immobilization and RI sensitivity also changes. So it is of great importance to study the RI sensing properties of surface immobilized nanostructures.

Even though RI sensitivity studies of different gold nanostructures are previously reported but there is a need for systematic study of anisotropic nanostructures with different sizes [10, 17, 18]. Evaluating the RI sensitivity of surface immobilized Au nanostructures is also very important for developing chipbased sensor systems. In this work, we report the synthesis of Au nanostars, nanocubes and nanorods by seed-mediated synthesis method. The size of the each nanostructure was systematically varied by changing the precursor concentrations. RI sensitivity of all three $\mathrm{Au}$ nanostructures with different sizes exhibiting LSPR peak positions in 500-900 nm spectral range were determined. This gives an idea about the RI sensitivity of different sizes for a given shape, which helps to choose the nanoparticle for a particular sensing platform. Samples exhibiting highest sensitivity in each category were immobilized on the glass substrate. The response of immobilized $\mathrm{Au}$ nanostructures to RI changes in the surrounding medium was studied and RI sensitivity was calculated. This study gives a broad outline for selecting $\mathrm{Au}$ nanostructures for developing nanoplasmonic sensing platforms by immobilizing the colloidal suspension of nanoparticles.

\section{Materials and methods}

\subsection{Chemical and reagents}

Cetyltrimethylammonium bromide (CTAB), silver nitrate $\left(\mathrm{AgNO}_{3}, 99.5 \%\right)$, sodium borohydride $\left(\mathrm{NaBH}_{4}\right)$, poly(sodium 4-styrenesulfonate) (PSSS, $1000 \mathrm{kDa}$ ) and (3-aminopropyl)trimethoxysilane (APTMS, 97\%) were procured from Sigma-Aldrich. Trisodium citrate dihydrate (99\%) and ascorbic acid (99\%) were purchased from Merck and Loba Chemie, respectively. Chloroauric acid and anhydrous 
glucose (99\%) were purchased from Alfa Aesar. All chemicals were used as received without any further purification. Deionized water from Milli-Q purification system was used for all synthesis.

\subsection{Synthesis of Au nanostructures}

\subsubsection{Au nanostars}

$\mathrm{Au}$ nanostars were synthesized using the previously reported surfactant free seed-mediated synthesis method with some modifications [19]. Initially to prepare seeds, $\mathrm{HAuCl}_{4}(1 \mathrm{mM}, 10 \mathrm{~mL})$ aqueous solution was boiled under stirring. To this trisodium citrate $(34 \mathrm{mM}, 1.5 \mathrm{~mL})$ aqueous solution was added and the stirring was continued. After $5 \mathrm{~min}$, the stirring was stopped, seed solution was cooled and stored at $4{ }^{\circ} \mathrm{C}$ for further use.

To prepare nanostars, aqueous solution of $\mathrm{HCl}$ $(1 \mathrm{M}, 10 \mu \mathrm{L})$ was added to $\mathrm{HAuCl}_{4}(10 \mathrm{~mL}, 0.25 \mathrm{mM})$ solution. Under continuous stirring $100 \mu \mathrm{L}$ of seed solution was added to this solution. Quickly, aqueous solution of $\mathrm{AgNO}_{3}(1 \mathrm{mM}, 100 \mu \mathrm{L})$ and ascorbic acid $(100 \mathrm{mM}, 50 \mu \mathrm{L})$ were added simultaneously and stirring was continued for a minute. Aqueous acetyl cysteine $(10 \mathrm{mM}, 10 \mu \mathrm{L})$ solution was immediately added and stirred for $5 \mathrm{~min}$. The Au nanostars colloidal solution was stored at room temperature in dark place.

\subsubsection{Au nanocubes}

Previously reported seed-mediated synthesis procedure was followed to prepare $\mathrm{Au}$ nanocubes [20]. For seed preparation, ice cold $\mathrm{NaBH}_{4}(0.6 \mathrm{~mL}, 100 \mathrm{mM})$ solution was added to aqueous solution of $\mathrm{HAuCl}_{4}$ $(10 \mathrm{~mL}, 0.25 \mathrm{mM})$ and CTAB $(75 \mathrm{mM})$ with stirring. After $5 \mathrm{~min}$ of stirring, the seed solution was kept undisturbed for $2 \mathrm{~h}$.

To get nanocubes, $25 \mathrm{~mL}$ of growth solution was prepared with $\mathrm{HAuCl}_{4}(0.2 \mathrm{mM})$ and CTAB $(16 \mathrm{mM})$. To this, aqueous ascorbic acid $(1.5 \mathrm{~mL}, 100 \mathrm{mM})$ solution was added with stirring. Finally different volumes (50-5 $\mu \mathrm{L})$ of 100 times diluted seed solutions were added. The stirring was stopped and the reaction was completed in 2-3 h.

\subsubsection{Au nanorods}

$\mathrm{Au}$ nanorods were synthesized with previously reported seed-mediated synthesis method [21]. To prepare seed solution, aqueous solution of $\mathrm{HAuCl}_{4}$ $(25 \mu \mathrm{L}, 50 \mathrm{mM})$ was added to CTAB solution $(5 \mathrm{~mL}$, $100 \mathrm{mM})$ with stirring. To this of $\mathrm{NaBH}_{4}(300 \mu \mathrm{L}$, $10 \mathrm{mM}$ ) aqueous solution was added quickly. Stirring was stopped after $5 \mathrm{~min}$.

The growth solution was prepared by combining following solutions to prepare $\mathrm{Au}$ nanorods. $\mathrm{HCl}(95$ $\mu \mathrm{L}, 1 \mathrm{M})$, CTAB $(5 \mathrm{~mL}, 100 \mathrm{mM}), \mathrm{HAuCl}_{4}(50 \mu \mathrm{L}$, $50 \mathrm{mM}$ ) solutions were combined and solution was shaken well. Next, different volumes of $\mathrm{AgNO}_{3}$ solution $(10-70 \mu \mathrm{L}, 10 \mathrm{mM})$, ascorbic acid $(50 \mu \mathrm{L}$, $100 \mathrm{mM}$ ) solution were added and shaken thoroughly. Finally $12 \mu \mathrm{L}$ of seed solution was added and the solution was kept undisturbed overnight for growth of nanorods.

Next, the colloidal solution was centrifuged at $5000 \mathrm{rpm}$ for $3 \mathrm{~min}$ to separate the spherical nanoparticles. After this, the supernatant solution was centrifuged at $7500 \mathrm{rpm}$ for $25 \mathrm{~min}$. The nanorods settled at the bottom were re-dispersed in DI water.

\subsection{Immobilization of $\mathrm{Au}$ nanostructures on glass surface}

To immobilize Au nanostars, piranha cleaned glass coverslips were immersed in $10 \%$ ethanolic solution of (3-aminopropyl)trimethoxysilane for $15 \mathrm{~min}$. Next, coverslips rinsed with deionized water and dried at $120{ }^{\circ} \mathrm{C}$ for $3 \mathrm{~h}$. After this, coverslips were incubated in gold nanostars solution and UV-Vis spectrum was recorded after different intervals of time.

To immobilize $\mathrm{Au}$ nanocubes and nanorods, piranha cleaned glass coverslips were immersed in $10 \%$ ethanolic solution for $1 \mathrm{~h}$ and dried at $120^{\circ} \mathrm{C}$ for $1 \mathrm{~h}$. After this, the coverslips were incubated in poly(sodium styrenesulfonate) (PSSS) solution (20 mg/ $\mathrm{mL}$ ) for one hour and then taken out and rinsed with water and were incubated in Au nanocubes colloidal solution. In case of $\mathrm{Au}$ nanorods, aqueous $\mathrm{NaCl}$ $(0.25 \mathrm{~mL}, 200 \mathrm{mM})$ solution was added to $2 \mathrm{~mL}$ of $\mathrm{Au}$ nanorods colloidal solution one hour prior to the incubation with silanized glass cover slips. UV-Vis spectrum was recorded after different intervals of incubation time. 


\subsection{Spectral and morphological characterization}

The spectral characterization of synthesized silver nanocubes was performed using lab-built UV-Vis spectroscopy setup explained in our earlier work $[22,23]$. All the LSPR experiments were also performed with this lab-built experimental setup.

All the FESEM images of surface immobilized $\mathrm{Au}$ nanostructures were recorded with Zeiss Sigma 300.

\subsection{Refractive index sensitivity measurements}

To measure the bulk RI sensitivity of Au nanostructures, various concentrations of aqueous solution of glucose $[0-20 \%(\mathrm{w} / \mathrm{v})]$ were used. Bulk RI sensitivity of all the samples were calculated by fitting the LSPR peak position versus RI of the surrounding medium plot. The bulk RI sensitivity of Au nanostructures immobilized on the glass substrate was also obtained by recoding the UV-Vis spectra of the LSPR chip in $0-40 \%$ aqueous glucose solution followed by fitting the plot of LSPR peak position against RI.

\section{Results and discussion}

\subsection{Synthesis of Au nanostructures}

All the Au nanostructures were prepared by seedmediated synthesis method. In the first step, seed nanoparticles were prepared and then small amount of these seeds were introduced into a growth medium which contains precursors in right proportions for formation of anisotropic nanostructures. By varying the concentration of the key precursors, nanostructures with a range of LSPR peak positions were obtained.

Surfactant free synthesis method was chosen to prepare Au nanostars, because the presence of layers of surfactants like $\mathrm{CTAB}$ on the nanostructures increases the distance between the surface of nanoparticles and analyte and hence reduce the RI sensitivity [24]. The seed particles were prepared by reducing the $\mathrm{Au}^{3+}$ ions with citrate at the water boiling temperature. The citrate protected seed particles were cooled to room temperature and further used in synthesis of Au nanostars. Different growth solutions were prepared with various amounts of
$\mathrm{AgNO}_{3}$ to get the $\mathrm{Au}$ nanostars with different LSPR peak positions. $\mathrm{Ag}^{+}$ions play crucial role in the formation of $\mathrm{Au}$ nanostars. Without $\mathrm{Ag}^{+}$ions, polydisperse nanoparticles were formed [19]. Ag do not form the branches on the $\mathrm{Au}$ nanocrystals but assist the formation of $\mathrm{Au}$ branches on certain crystallographic facets of multi-twinned $\mathrm{Au}$ seeds. Further, the final concentration of $\mathrm{AgNO}_{3}$ in growth solution is in 5-25 $\mu \mathrm{M}$ range which is very less compared to the concentration of $\mathrm{HAuCl}_{4}$ which is $250 \mu \mathrm{M}$. Ag may constitute $2-4.5 \%$ of Au branches [19]. Au nanostars samples with LSPR maxima positioned from 664 to $868 \mathrm{~nm}$ were obtained by increasing the concentration $\mathrm{AgNO}_{3}$ from 0.5 to $2.5 \mathrm{mM}$ while preparing the growth solution (Fig. 1a). In CTAB-assisted synthesis method of $\mathrm{Au}$ nanostars, a bilayer of CTAB formed on the surface effectively protects the nanostars. Au nanostars obtained by this surfactant free method were found to be unstable, $35 \mathrm{~nm}$ blue shift in LSPR peak position was observed after one day. This indicates the degradation of nanostructures. To prevent this, small amount of acetyl cysteine $(10 \mathrm{mM}, 10$ $\mu \mathrm{L})$ was added. This effectively stabilized the $\mathrm{Au}$ nanostars and not much considerable shift in LSPR peak position was observed even after 8 days. It can be hypothesized acetyl cysteine binds to the surface of nanoparticles forming the $\mathrm{Au}-\mathrm{S}$ bond and hence protect and stabilize the Au nanostars [25].

For the synthesis of $\mathrm{Au}$ nanocubes and nanorods, we followed the surfactant-assisted seed-mediated synthesis method. For these nanostructures, CTAB surfactant plays crucial role in inducing the shape and CATB-assisted method is highly reliable and gives good yield [26]. To synthesize Au nanocubes, seed particles were prepared by reducing the $\mathrm{Au}^{3+}$ ions with $\mathrm{NaBH}_{4}$ in the presence of CTAB. Aqueous growth solution containing $\mathrm{CTAB}, \mathrm{HAuCl}_{4}$, and ascorbic acid was prepared and different volumes of seed solutions were introduced. Within $2-3 \mathrm{~h}$ the growth solution showed stable color (pink to blue, depending on the seed solution volume). This indicated the completion of the formation of nanocubes. $\mathrm{CTAB}$ and $\mathrm{Au}^{+}$ions were initially mixed in growth solution to form $\mathrm{AuBr}_{4}{ }^{-}$complex. Next the complex was reduced to $\mathrm{Au}^{+}$by ascorbic acid. Ascorbic acid is a mild reducing agent and cannot reduce $\mathrm{Au}^{3+}$ into $\mathrm{Au}^{0}$ in the presence of CTAB micelle without the presence of seed [27]. Next, different volumes of seed solutions were introduced into this growth medium. The seeds increase the growth rate of particles and in 

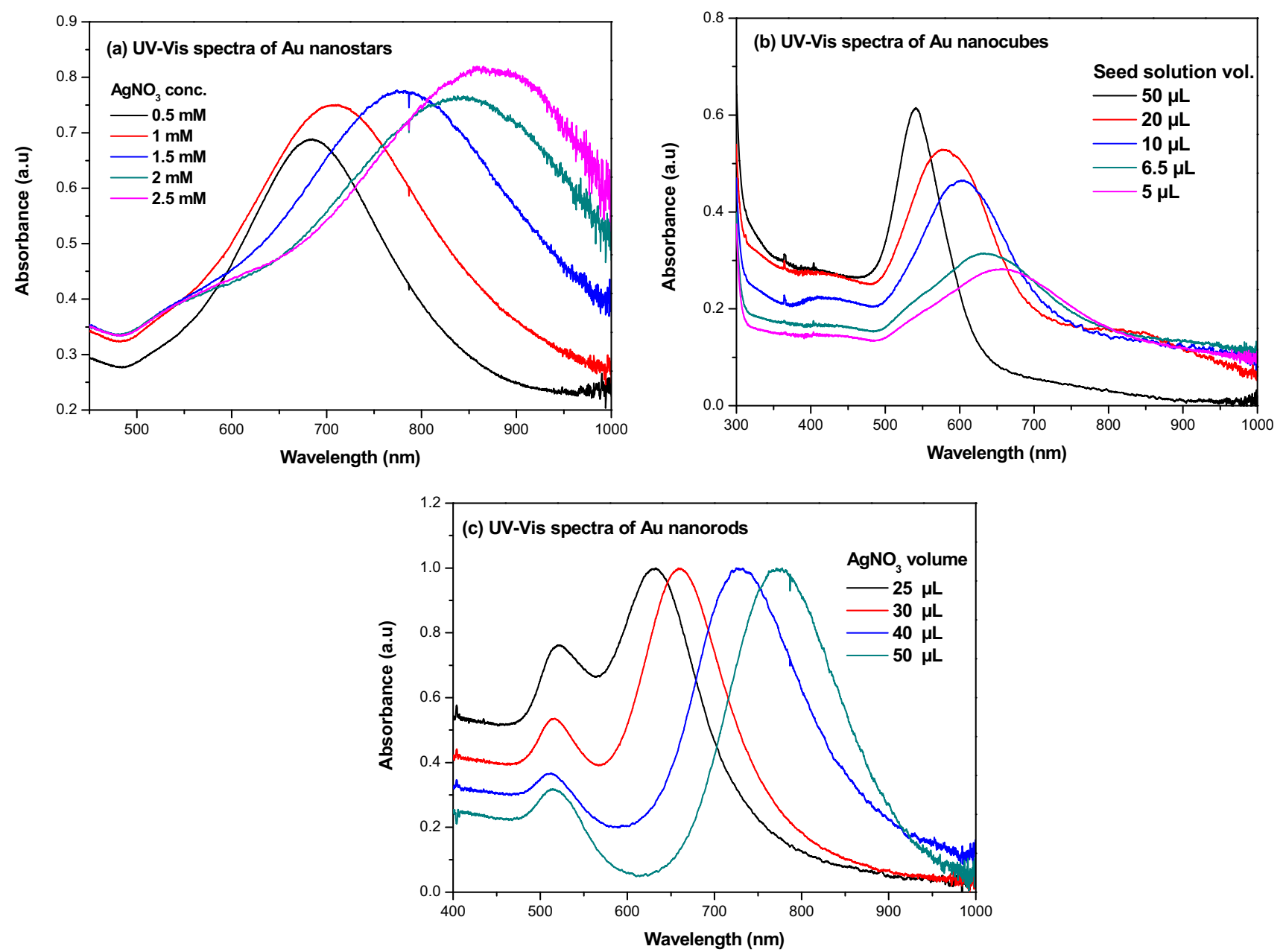

Fig. 1 UV-Vis absorption spectra of a Au nanostars synthesized with 0.5, 1, 1.5, 2, $2.5 \mathrm{mM} \mathrm{AgNO}$ solution. b Au nanocubes synthesized with $50,20,10,6.5,5 \mu \mathrm{L}$ of seed solution. c Au nanorods prepared with $25,30,40,50 \mu \mathrm{L}$ of $\mathrm{AgNO}_{3}$ solution

the absence of seeds, no color change can be observed. No other reagents were used to induce the shape. The amount of seeds added to the growth medium determines the size of the Au nanocubes, the seed solution was 100 times diluted with deionized water before adding to the growth solution [20]. Addition of 50, 20, 10, 6.5 and $5 \mu \mathrm{L}$ of diluted seed solutions to the growth solution resulted in the formation of $\mathrm{Au}$ nanocubes with LSPR peak positions at 536, 575, 595, 625 and $647 \mathrm{~nm}$ (Fig. 1b). This shows that, decrease in the number of seed particles available in the growth medium resulted in formation of larger nanocubes. With increase in number of seed particles the number of $\mathrm{Au}$ atoms available per seed particle decreases and hence the final size of the nanocubes is smaller. This observation is consistent with the previous reports pertaining to other plasmonic nanostructures [22, 23].
Seed-mediated growth method is the most reliable and effective method of preparation of Au nanorods. $\mathrm{CTAB}$ capped seeds were introduced to the growth solution containing $\mathrm{HCl}, \mathrm{CTAB}, \mathrm{HAuCl}_{4}, \mathrm{AgNO}_{3}$ and ascorbic acid. Figure 1c shows the UV-Vis spectra of $\mathrm{Au}$ nanorods synthesized with different amounts of $\mathrm{AgNO}_{3}$ solution to the growth medium. As in case of $\mathrm{Au}$ nanocubes, initially $\mathrm{CTAB}$ and $\mathrm{HAuCl}_{4}$ solutions were combined to form $\mathrm{AuBr}_{4}{ }^{-}$complex. By adding ascorbic acid, complex was reduced to $\mathrm{Au}^{+}$. Before introducing the seed solution, $\mathrm{AgNO}_{3}$ solution was added to the growth medium. For formation and controlling of aspect ratio of $\mathrm{Au}$ nanorods, presence of $\mathrm{Ag}^{+}$ions is essential [27]. It is previously reported that, the aspect ratio of nanorods could not be controlled by varying the seed to metal ion ratio in the absence of $\mathrm{Ag}^{+}$ions [27]. When the seeds were introduced to the growth medium, the rod formation 
was started and the growth rate was reduced by lower $\mathrm{pH}$, which was achieved by adding $\mathrm{HCl}$ while preparing the growth solution [21]. Slow growth helps the formation of good quality nanorods. Nanorods show two plasmonic bands due to transverse and longitudinal plasmon resonance. The transverse resonance band appears around $520 \mathrm{~nm}$ and longitudinal resonance band varies from $600 \mathrm{~nm}$ to NIR region depending on the aspect ratio [28]. With increase in the aspect ratio, longitudinal resonance band moves towards higher wavelength region. In our case, longitudinal plasmon resonance band moved from 631 to $774 \mathrm{~nm}$ with increase in the volume of $\mathrm{AgNO}_{3}$ solution from 25 to $50 \mu \mathrm{L}$ and the transverse plasmon resonance band almost remained in the $\sim 515 \mathrm{~nm}$. This indicates the formation of higher aspect ratio nanorods with increase in the concentration of $\mathrm{AgNO}_{3}$ in the growth medium [29]. $\mathrm{Au}$ nanorods were purified by two times centrifugation. This helped to remove maximum amount of spherical and other small nanoparticles and improved the quality of Au nanorods [30].

\subsection{Refractive index sensitivity of Au nanostructures}

When natural oscillation frequency of free electrons confined to the metal nanostructures matches with the frequency of incident electromagnetic radiation the LSPR phenomenon is observed. Resonance conditions can be deduced for small spherical nanoparticles using discrete dipole approximation and frequency-dependent dielectric function from Drude model. The resonance condition is expressed as $\varepsilon_{1-}$ $(\omega)=-2 \varepsilon_{\mathrm{d}}$, where $\varepsilon_{1}$ is the real part of dielectric function of metal, $\omega$ is the frequency of incident light and $\varepsilon_{\mathrm{d}}$ is the dielectric function of surrounding medium [31]. By taking the resonance condition into account, the LSPR peak position $\left(\lambda_{\max }\right)$ can be expressed as $\lambda_{\max }=\lambda_{\mathrm{p}}\left(2 n_{\mathrm{d}}{ }^{2}+1\right)^{1 / 2}$, where $\lambda_{\mathrm{p}}$ is the plasma wavelength of the metal and $n_{\mathrm{d}}{ }^{2}=\varepsilon_{\mathrm{d}}$. This relationship can be approximately written as $\lambda_{\max }=$ $\sqrt{2} \lambda_{\mathrm{p}} * n_{\mathrm{d}}$ in visible region of electromagnetic spectrum [31, 32]. This indicates that within a sufficiently narrow range of $n_{\mathrm{d}}$, a plot of $\lambda_{\max }$ versus $n_{\mathrm{d}}$ must be linear. This forms the basis for calculating RI sensitivity of plasmonic nanostructures. In case of anisotropic nanostructures it is not possible to use discrete approximation but numerical computational methods can be used.
Experimental assessment of RI sensitivity of the plasmonic nanostructures is the first step in the selection of nanostructures for fabrication of LSPRbased refractometric sensors. Here we gradually increased the RI by increasing the glucose concentration from 0 to $20 \%$ in steps of $5 \%$. After each step, UV-Vis spectrum was recorded and the process was repeated with different batches of all the three $\mathrm{Au}$ nanostructures. In Fig. 2, response to the RI change of $\mathrm{Au}$ nanostructures with highest RI sensitivity are shown. Successive redshift in LSPR peak position can be seen in Fig. 2a, c, e with increase in RI of the surrounding medium. The slope of the plot of LSPR peak position vs. RI gives the RI sensitivity of a particular nanostructure. Figure $2 b, d, f$ show the linearly fitted plot of LSPR peak position vs. RI of the surrounding medium. The LSPR peak positions were obtained by finding the zero crossing position of linearly fitted derivative of small spectral region close to the absorption maximum. RI sensitivity of all the samples in the solution were calculated and the values are tabulated in Table 1. Figure $3 a-c$ graphically show the variation of RI sensitivity with increasing LSPR peak position for Au nanostars, nanocubes and nanorods. It can be observed that RI sensitivities of $\mathrm{Au}$ nanostructures increase with redshift in LSPR peak position to certain extent and then decrease. Au nanostars, nanocubes and nanorods with LSPR peak positions at 767, 596 and $732 \mathrm{~nm}$ exhibited highest RI sensitivity of 484, 184 and 317, respectively. Similar trend was observed in our previous reports in case of Ag triangular nanoplates [22, 23]. For Au nanocubes with LSPR peak position at $647 \mathrm{~nm}$ showed increased sensitivity but it is still less compared to RI sensitivity of $\mathrm{Au}$ nanocubes with LSPR maxima at $596 \mathrm{~nm}$. Further experiments were carried out to prepare $\mathrm{Au}$ nanocubes with longer LSPR maximum wavelength but well-defined UV-Vis spectrum was not observed. All these Au nanostructures with highest RI sensitivity were selected for immobilization for fabrication of the LSPR chip.

\subsection{Immobilization of Au nanostructures}

To immobilize $\mathrm{Au}$ nanostars, glass coverslips were cleaned with piranha solution $\left(\mathrm{H}_{2} \mathrm{SO}_{4}: \mathrm{H}_{2} \mathrm{O}_{2}, 3: 1 \mathrm{v} / \mathrm{v}\right)$ to remove all the organic impurities present on the substrate. APTMS molecular layer was formed on the substrate by incubating the cleaned cover slip in 15\% ethanolic solution of APTMS. After $15 \mathrm{~min}$ of 

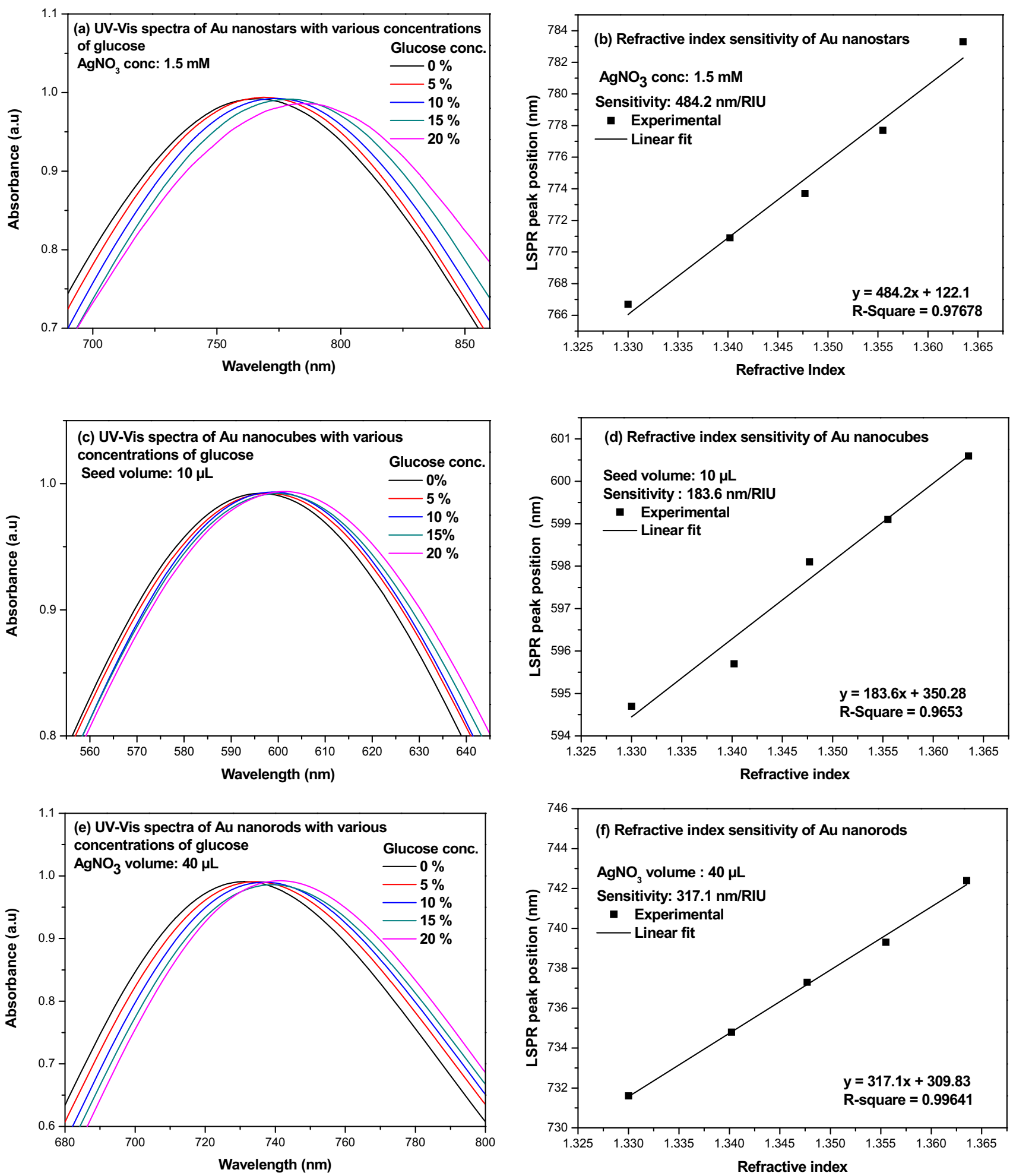

Fig. 2 UV-Vis spectra showing redshift in LSPR peak position of a Au nanostars, c Au nanocubes, and e Au nanorods with increase in glucose concentration from 0 to $20 \%$. Linearly fitted plot of

LSPR peak position vs. refractive index for $\mathbf{b} \mathrm{Au}$ nanostars, $\mathbf{d} \mathrm{Au}$ nanocubes and $\mathbf{f} \mathrm{Au}$ nanorods 
Table 1 RI sensitivities of Au nanostructures obtained with glucose refractive index sensitivity analysis

\begin{tabular}{|c|c|c|c|c|c|}
\hline \multicolumn{2}{|l|}{ Au nanostars } & \multicolumn{2}{|l|}{ Au nanocubes } & \multicolumn{2}{|l|}{ Au nanorods } \\
\hline $\begin{array}{l}\text { LSPR peak position } \\
(\mathrm{nm})\end{array}$ & $\begin{array}{l}\text { RI sensitivity (nm/ } \\
\text { RIU) }\end{array}$ & $\begin{array}{l}\text { LSPR peak position } \\
(\mathrm{nm})\end{array}$ & $\begin{array}{l}\text { RI sensitivity (nm/ } \\
\text { RIU) }\end{array}$ & $\begin{array}{l}\text { LSPR peak position } \\
(\mathrm{nm})\end{array}$ & $\begin{array}{l}\text { RI sensitivity (nm/ } \\
\text { RIU) }\end{array}$ \\
\hline 664 & $289 \pm 12$ & 536 & $47 \pm 8$ & 631 & $164 \pm 8$ \\
\hline 734 & $419 \pm 14$ & 575 & $106 \pm 6$ & 661 & $170 \pm 6$ \\
\hline 767 & $484 \pm 12$ & 596 & $184 \pm 8$ & 732 & $317 \pm 8$ \\
\hline 847 & $419 \pm 10$ & 625 & $121 \pm 10$ & 774 & $280 \pm 5$ \\
\hline 868 & $401 \pm 14$ & 647 & $158 \pm 8$ & - & - \\
\hline
\end{tabular}
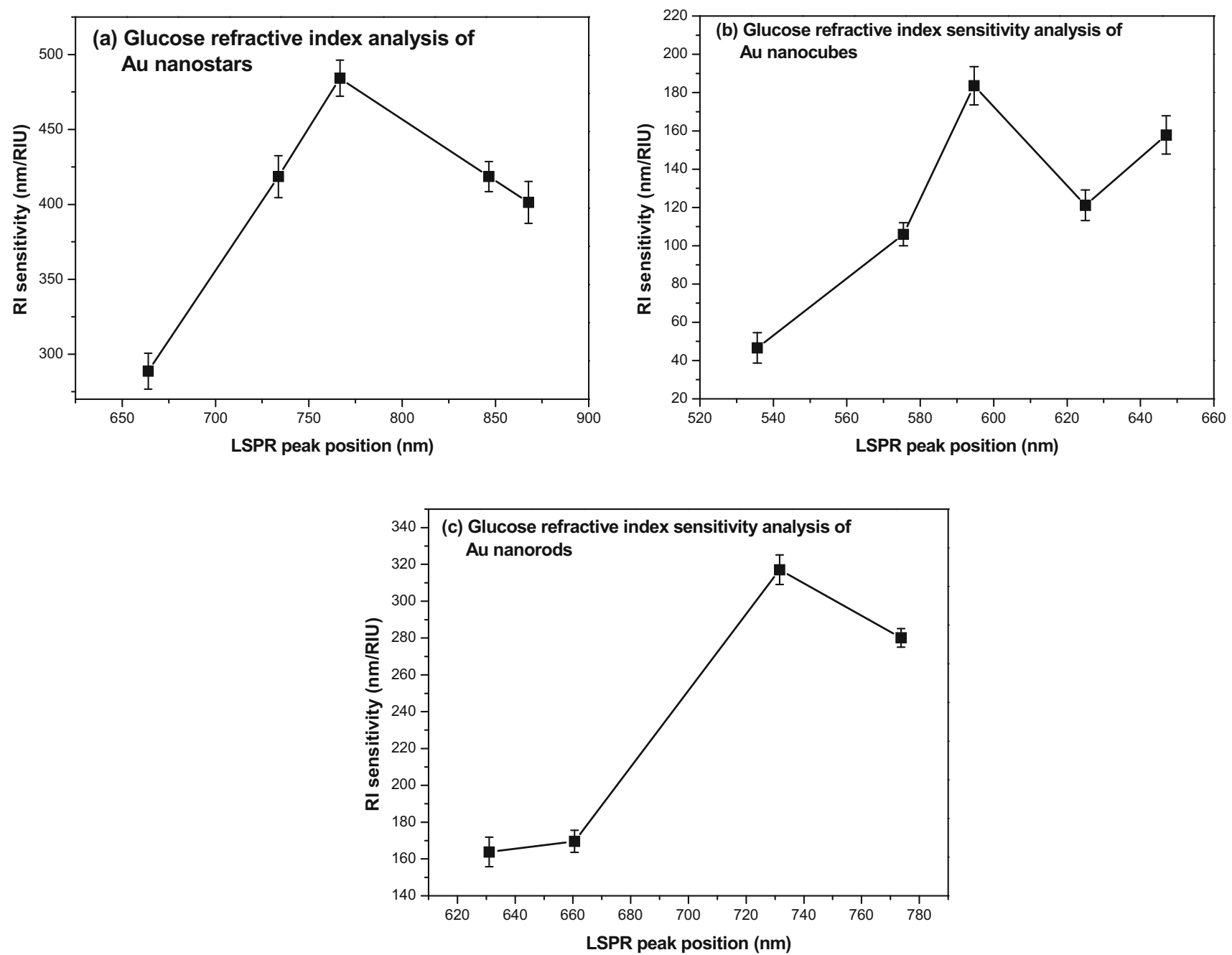

Fig. 3 Variation of refractive index sensitivity with LSPR peak position for a Au nanostars, $\mathbf{b}$ Au nanocubes and $\mathbf{c}$ Au nanorods

incubation, the coverslips were washed and dried at $120{ }^{\circ} \mathrm{C}$ for $3 \mathrm{~h}$ to minimize the multilayer formation. These coverslips were incubated in $\mathrm{Au}$ nanostars colloidal solution and UV-Vis spectrum was recorded after different intervals of time. The amount of nanostars immobilization on the substrate was controlled by incubation time. After $40 \mathrm{~h}$ of incubation, the absorbance was almost remained same as shown in Fig. 4a. To immobilize Au nanocubes and nanorods the procedure was modified due to the presence 

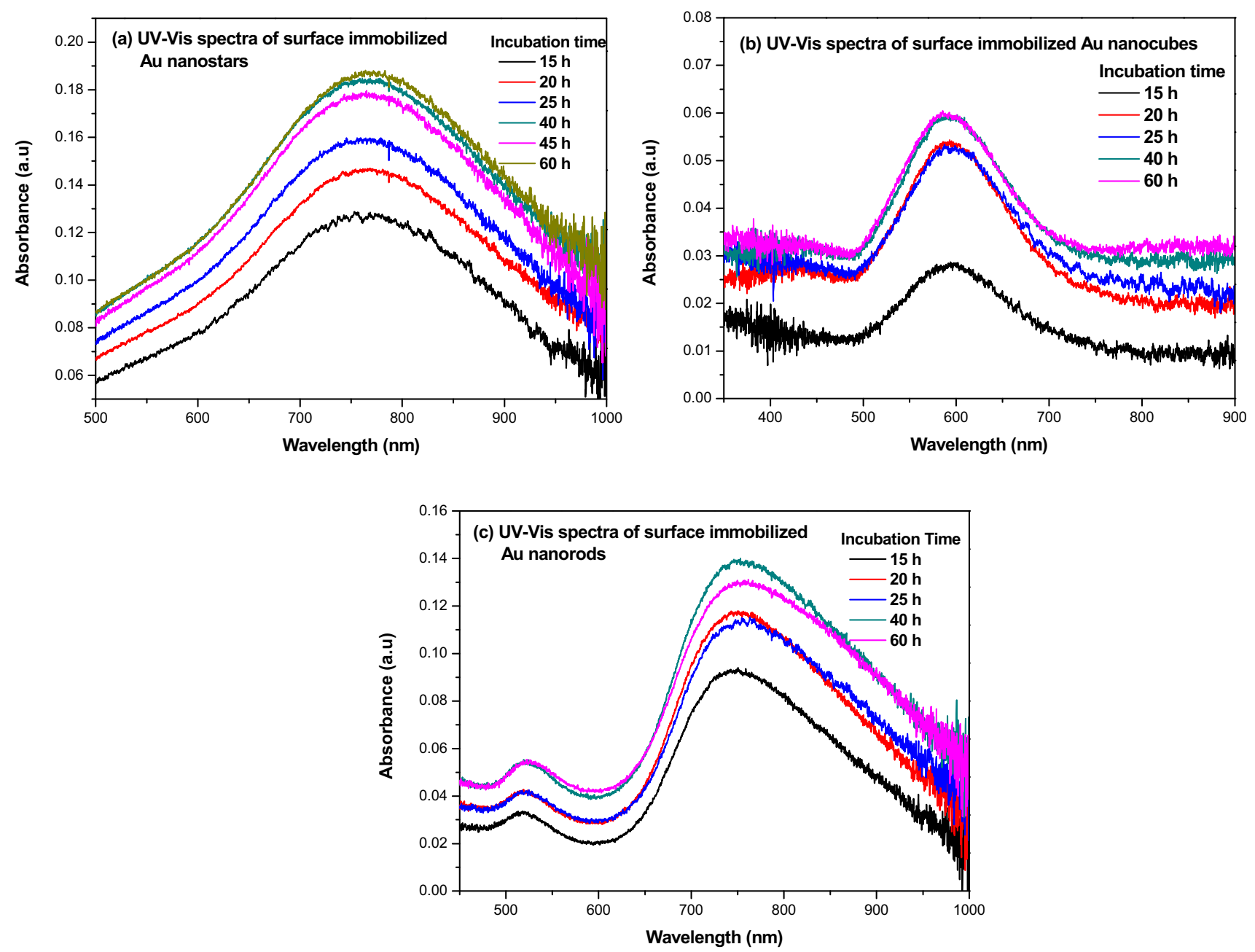

Fig. 4 UV-Vis spectra of surface immobilized a Au nanostars, b Au nanocubes, and c Au nanorods recorded after different intervals of incubation time $(15-60 \mathrm{~h})$

CTAB surfactant on the surface of these nanostructures [33]. APTMS modified glass coverslips were incubated with PSSS solution in order to form the negatively charged layer on the surface. Positively charged $\mathrm{CTAB}$ capped gold nanostructures can electrostatically adhere to the surface modified glass coverslip. In case of gold nanocubes, the UV-Vis spectra recorded after different incubation time period is shown in Fig. 4b. For immobilization of $\mathrm{Au}$ nanorods, $\mathrm{NaCl}$ was introduced into the nanorods colloidal solution which helps to form the electrostatic-based dense surface assembly of Au nanorods on the APTMS-PSSS modified glass coverslip [34]. Figure 4c shows the UV-Vis spectra of surface immobilized $\mathrm{Au}$ nanorods recorded after different intervals of incubation time and both transverse and longitudinal plasmon resonance band can be clearly observed. Red shift is observed after immobilization of $\mathrm{Au}$ nanostars and nanorods and a small blue shift in case of $\mathrm{Au}$ nanocubes (Table 2). The mode of coupling between the nanoparticles induce a redshift or blue shift in the LSPR band [35]. The surface immobilized $\mathrm{Au}$ nanostructures also show all the plasmonic bands which are observed in the colloidal solution and no additional bands can be observed. This indicates that all the nanostructures are immobilized on the substrate without sacrificing their characteristic features. Figure 5 shows FESEM images of all surface immobilized nanostructures which indicates the successful immobilization of $\mathrm{Au}$ nanostructures on the silanized glass substrate. The average sizes of $\mathrm{Au}$ nanostars and $\mathrm{Au}$ nanocubes are found to be 73 and $85 \mathrm{~nm}$. The average aspect ratio of $\mathrm{Au}$ nanorods is 2.7 . 
Table 2 LSPR peak positions of Au nanostructures in solution and on the substrate

\begin{tabular}{llllll}
\hline Nanostructure & \multicolumn{2}{l}{ Solution } & & \multicolumn{2}{l}{ Substrate } \\
\cline { 2 - 3 } & LSPR peak position $(\mathrm{nm})$ & RI sensitivity $(\mathrm{nm} / \mathrm{RIU})$ & & LSPR peak position $(\mathrm{nm})$ & RI sensitivity (nm/RIU) \\
\hline Au nanostars & 767 & $484 \pm 12$ & 773 & $318 \pm 10$ \\
Au nanocubes & 596 & $184 \pm 8$ & 593 & $95 \pm 8$ \\
Au nanorods & 732 & $317 \pm 8$ & 753 & $108 \pm 10$ \\
\hline
\end{tabular}
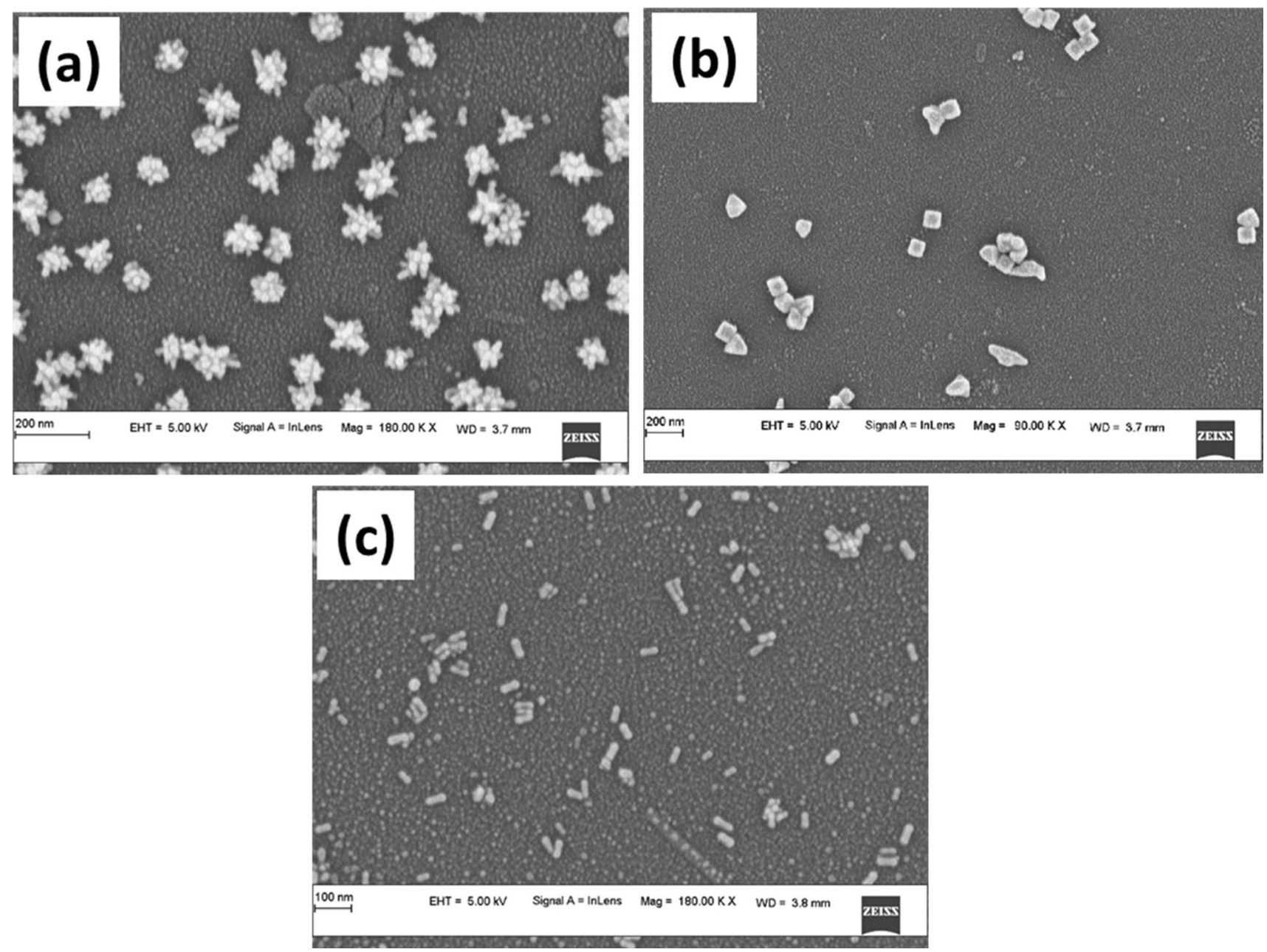

Fig. 5 FESEM images of a Au nanostars (average size $73 \mathrm{~nm}$ ), b Au nanocubes (average size $85 \mathrm{~nm}$ ), and c Au nanorods (average aspect ratio 2.7) immobilized on the silanized glass substrate

To check the response of surface immobilized Au nanostructures to the RI modulation, UV-Vis spectra of those coverslips were recorded in air and in water. When the coverslips are transferred from air to water, the RI of the surrounding medium changes from 1 to 1.33. Large redshift in LSPR peak position can be expected due to the drastic change in RI of the surrounding medium. The UV-Vis spectra of all surface immobilized nanostructures show clear redshift in LSPR peak position when the medium changes from air to water (Fig. 6a-c). The spectra were recorded several times to check the reversibility of the plasmon band position. Every time the LSPR bands of all the nanostructures restored their positions, which indicates that the surface immobilized Au nanostructures 

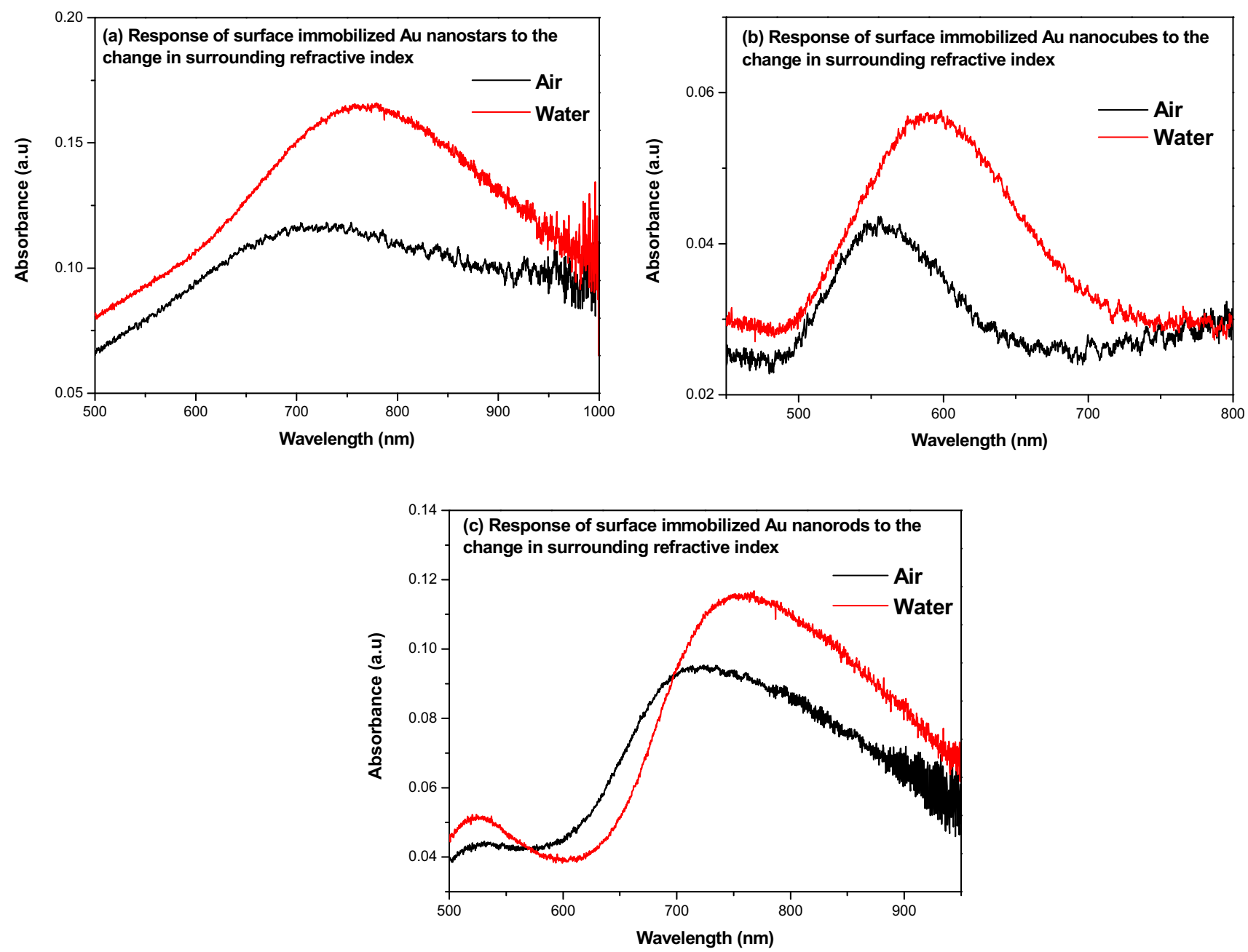

Fig. 6 UV-Vis spectra of surface immobilized a Au nanostars, b Au nanocubes, and c Au nanorods in air and in water

are highly reliable for refractometric sensing applications.

\subsection{Refractive index sensitivity of immobilized Au nanostructures}

After immobilization, RI sensitivities of all the Au nanostructures were determined with different concentrations of glucose solution. Glass coverslips with $\mathrm{Au}$ nanostructures were immersed in $0-40 \%$ glucose solutions and UV-Vis spectra were recorded with each solution. Figure $7 \mathrm{a}, \mathrm{c}$, e show the response of plasmonic band of Au nanostructures to the changing glucose concentration. As in case of colloidal solutions, LSPR band maxima were determined for all the nanostructures. The slope of the linear fit of LSPR peak position vs. RI gives the RI sensitivity of corresponding surface immobilized $\mathrm{Au}$ nanostructure
(Fig. $7 b, d, f)$. Table 2 lists the RI sensitivity of $\mathrm{Au}$ nanostars, nanocubes and nanorods in solution and on the substrate. RI sensitivity of $\mathrm{Au}$ nanostars is decreased by $34 \%$, Au nanocubes is decreased by $48 \%$ and $\mathrm{Au}$ nanorods is decreased by $66 \%$ after immobilization on the glass substrate. Strong correlation is reported between the RI sensitivity and fraction of the surface which is in direct contact with the substrate [16]. The effective RI around the nanostructures changes after immobilization. Here the decrease in the RI sensitivity can be attributed to the interaction between substrate and nanostructures. From this study we can infer that $\mathrm{Au}$ nanostars show higher sensitivity compared to Au nanocubes and nanorods both in solution and on the substrate. 

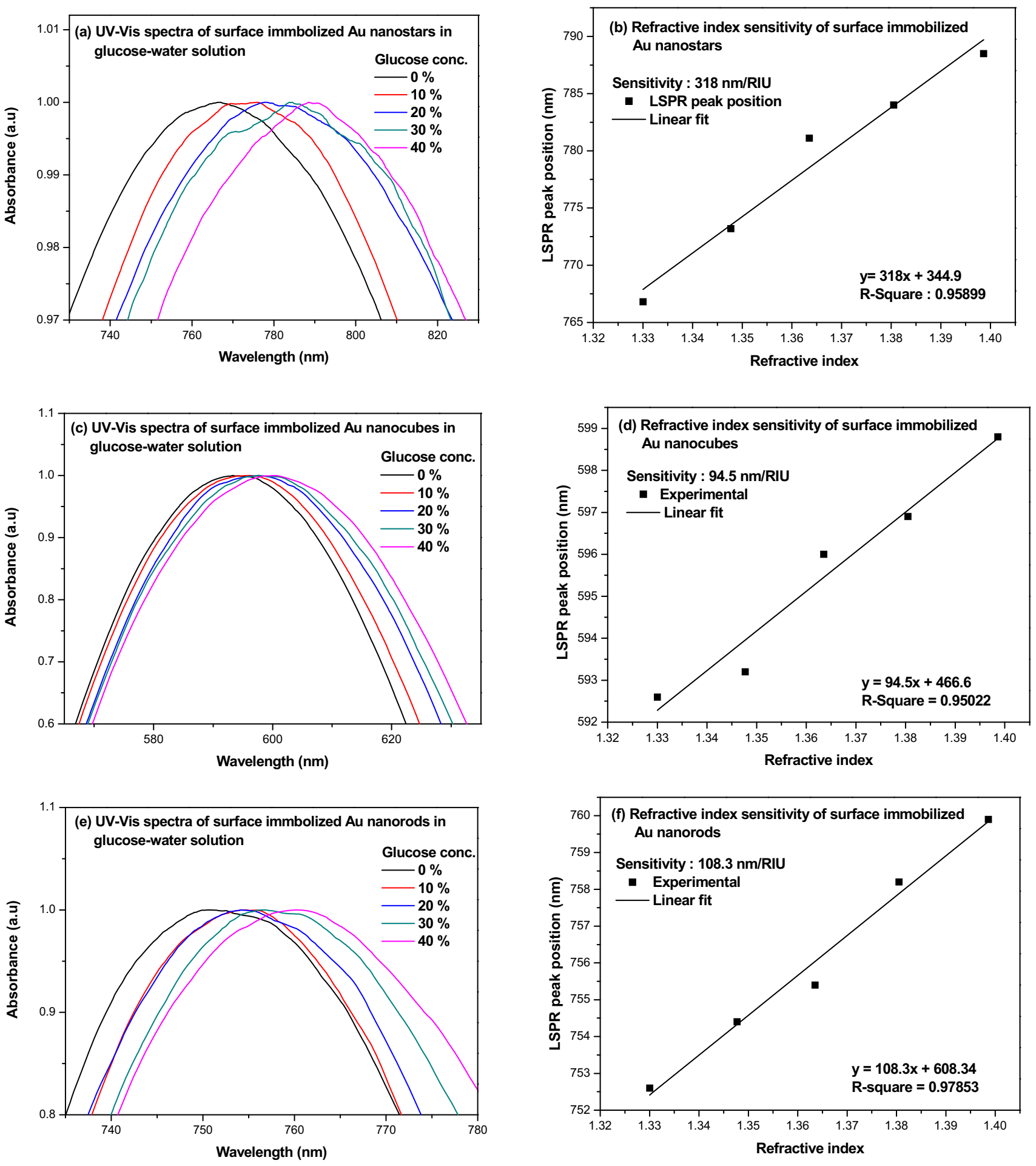

Fig. 7 UV-Vis spectra showing redshift in LSPR peak position of surface immobilized a Au nanostars, c Au nanocubes, and e Au nanorods with increase in glucose concentration from 0 to $20 \%$.

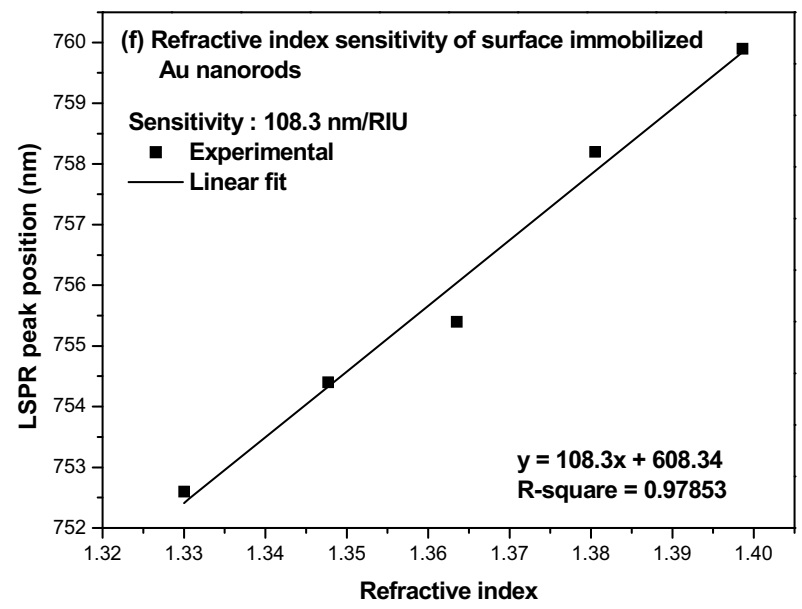

Linearly fitted plot of LSPR peak position vs. refractive index for surface immobilized $\mathbf{b} \mathrm{Au}$ nanostars, $\mathbf{d} \mathrm{Au}$ nanocubes, and $\mathbf{f} \mathrm{Au}$ nanorods 


\section{Conclusions}

We have synthesized Au nanostars, nanocubes and nanorods by seed-mediated synthesis method with LSPR peak positions varying in the range of 500-900 nm. RI sensitivity of all these nanostructures were determined in the solution and the sample with highest RI sensitivity in each category were immobilized on the glass substrate. Response of surface immobilized Au nanostructures to the RI modulation was checked and RI sensitivity measurements were performed. Au nanostars with $73 \mathrm{~nm}$ average size and LSPR peak position at $767 \mathrm{~nm}$ exhibited highest RI sensitivity of $484 \mathrm{~nm} /$ RIU in solution and $318 \mathrm{~nm} /$ RIU on the substrate. This RI sensitivity investigation of $\mathrm{Au}$ nanostructures gives an outline for selecting nanostructures for development of the nanoplasmonic sensing platforms.

\section{Acknowledgements}

Financial support from Department of Science and Technology (DST), India under the Project Grant No. IDP/BDTD/11/2019 is gratefully acknowledged. Hemant Ramakant Hegde acknowledges the TMA Pai PhD Fellowship from Manipal Academy of Higher Education (MAHE).

\section{Author contributions}

HRH performed all the experimental work, collected and analyzed the data and wrote the first draft of the manuscript. Approved the final version of the manuscript. SC revised the manuscript and approved the final version of the manuscript. RKS conceptualized and designed the work, revised the manuscript and approved the final version of the manuscript.

\section{Funding}

Open access funding provided by Manipal Academy of Higher Education, Manipal.

\section{Data availability}

The datasets generated during and/or analyzed during the current study are available from the corresponding author on reasonable request.

\section{Declarations}

Conflict of interest Authors declare no conflicts of interest.

Open Access This article is licensed under a Creative Commons Attribution 4.0 International License, which permits use, sharing, adaptation, distribution and reproduction in any medium or format, as long as you give appropriate credit to the original author(s) and the source, provide a link to the Creative Commons licence, and indicate if changes were made. The images or other third party material in this article are included in the article's Creative Commons licence, unless indicated otherwise in a credit line to the material. If material is not included in the article's Creative Commons licence and your intended use is not permitted by statutory regulation or exceeds the permitted use, you will need to obtain permission directly from the copyright holder. To view a copy of this licence, visit http://creativecommons.org/licen ses/by/4.0/.

\section{References}

1. E. Petryayeva, U.J. Krull, Localized surface plasmon resonance: nanostructures, bioassays and biosensing - a review. Anal. Chim. Acta 706(1), 8-24 (2011)

2. G.V. Hartland, Optical studies of dynamics in noble metal nanostructures. Chem. Rev. 111(6), 3858-3887 (2011)

3. E. Martinsson, D. Aili, Refractometric sensing using plasmonic nanoparticles, in Encyclopedia of Nanotechnology. ed. by B. Bhushan (Springer, Dordrecht, 2016), pp. 3432-3440

4. L.M. Liz-Marzán, Tailoring surface plasmons through the morphology and assembly of metal nanoparticles. Langmuir 22(1), 32-41 (2006)

5. H.R. Hegde, S. Chidangil, R.K. Sinha, Review of synthesis and sensing applications of anisotropic silver and gold nanostructures. J. Vac. Sci. Technol. B 39(5), 050801 (2021)

6. A.R. Tao, S. Habas, P. Yang, Shape control of colloidal metal nanocrystals. Small 4(3), 310-325 (2008)

7. K.M. Mayer, J.H. Hafner, Localized surface plasmon resonance sensors. Chem. Rev. 111(6), 3828-3857 (2011) 
8. Y. Chen, Y. Xianyu, X. Jiang, Surface modification of gold nanoparticles with small molecules for biochemical analysis. Acc. Chem. Res. 50(2), 310-319 (2017)

9. C. Shen et al., Site-specific surface functionalization of gold nanorods using DNA Origami clamps. J. Am. Chem. Soc. 138(6), 1764-1767 (2016)

10. H. Chen et al., Shape- and size-dependent refractive index sensitivity of gold nanoparticles. Langmuir 24(10), 5233-5237 (2008)

11. S. Barbosa et al., Tuning size and sensing properties in colloidal gold nanostars. Langmuir 26(18), 14943-14950 (2010)

12. S.M. Marinakos, S. Chen, A. Chilkoti, Plasmonic detection of a model analyte in serum by a gold nanorod sensor. Anal. Chem. 79(14), 5278-5283 (2007)

13. P. Tuersun et al., Refractive index sensitivity analysis of gold nanoparticles. Optik 149, 384-390 (2017)

14. M.M. Miller, A.A. Lazarides, Sensitivity of metal nanoparticle surface plasmon resonance to the dielectric environment. J. Phys. Chem. B 109(46), 21556-21565 (2005)

15. H.B. Jeon, P.V. Tsalu, J.W. Ha, Shape effect on the refractive index sensitivity at localized surface plasmon resonance inflection points of single gold nanocubes with vertices. Sci. Rep. 9(1), 13635 (2019)

16. E. Martinsson et al., Substrate effect on the refractive index sensitivity of silver nanoparticles. J. Phys. Chem. C 118(42), 24680-24687 (2014)

17. H. Chen et al., Shape-dependent refractive index sensitivities of gold nanocrystals with the same plasmon resonance wavelength. J. Phys. Chem. C 113(41), 17691-17697 (2009)

18. C.L. Nehl, H. Liao, J.H. Hafner, Optical properties of starshaped gold nanoparticles. Nano Lett. 6(4), 683-688 (2006)

19. H. Yuan et al., Gold nanostars: surfactant-free synthesis, 3D modelling, and two-photon photoluminescence imaging. Nanotechnology 23(7), 075102 (2012)

20. J.J. Sheng et al., A preliminary study on surface-enhanced Raman scattering from Au and Au@Pd nanocubes for electrochemical applications. Can. J. Anal. Sci. Spectrosc. 52, 178-185 (2007)

21. L. Scarabelli et al., A “Tips and Tricks" practical guide to the synthesis of gold nanorods. J. Phys. Chem. Lett. 6(21), 4270-4279 (2015)

22. H. Hegde, C. Santhosh, R.K. Sinha, Seed mediated synthesis of highly stable CTAB capped triangular silver nanoplates for LSPR sensing. Mater. Res. Express 6(10), 105075 (2019)

23. H.R. Hegde, S. Chidangil, R.K. Sinha, Refractive index sensitivity of triangular $\mathrm{Ag}$ nanoplates in solution and on glass substrate. Sens. Actuators A 305, 111948 (2020)
24. J. Otsuki, K. Sugawa, S. Jin, Plasmonic triangular nanoprism sensors. Mater. Adv. 2(1), 32-46 (2021)

25. L. Zhang et al., Self-assembly of L-cysteine-gold nanoparticles as chiral probes for visual recognition of 3,4-dihydroxyphenylalanine enantiomers. RSC Adv. 5(34), 27003-27008 (2015)

26. T.K. Sau, C.J. Murphy, Room temperature, high-yield synthesis of multiple shapes of gold nanoparticles in aqueous solution. J. Am. Chem. Soc. 126(28), 8648-8649 (2004)

27. N.R. Jana, L. Gearheart, C.J. Murphy, Seed-mediated growth approach for shape-controlled synthesis of spheroidal and rod-like gold nanoparticles using a surfactant template. Adv. Mater. 13(18), 1389-1393 (2001)

28. J. Pérez-Juste et al., Gold nanorods: synthesis, characterization and applications. Coord. Chem. Rev. 249(17), 1870-1901 (2005)

29. W. Tong et al., Control of symmetry breaking size and aspect ratio in gold nanorods: underlying role of silver nitrate. J. Phys. Chem. C 121(6), 3549-3559 (2017)

30. M. Boksebeld et al., Shape-selective purification of gold nanorods with low aspect ratio using a simple centrifugation method. Gold Bull. 50(1), 69-76 (2017)

31. J. Langer, S.M. Novikov, L.M. Liz-Marzán, Sensing using plasmonic nanostructures and nanoparticles. Nanotechnology 26(32), 322001 (2015)

32. T.R. Jensen et al., Nanosphere lithography: effect of the external dielectric medium on the surface plasmon resonance spectrum of a periodic array of silver nanoparticles. J. Phys. Chem. B 103(45), 9846-9853 (1999)

33. J.A. Harrington, Z.D. Harms, J.M. Zaleski, Electrostatic assembly of gold nanorods on a glass substrate for sustainable photocatalytic reduction via sodium borohydride. RSC Adv. 6(64), 59113-59123 (2016)

34. A.R. Ferhan, L. Guo, D.-H. Kim, Influence of ionic strength and surfactant concentration on electrostatic surfacial assembly of cetyltrimethylammonium bromide-capped gold nanorods on fully immersed glass. Langmuir 26(14), 12433-12442 (2010)

35. P.K. Jain, S. Eustis, M.A. El-Sayed, Plasmon coupling in nanorod assemblies: optical absorption, discrete dipole approximation simulation, and exciton-coupling model. J. Phys. Chem. B 110(37), 18243-18253 (2006)

Publisher's Note Springer Nature remains neutral with regard to jurisdictional claims in published maps and institutional affiliations. 